\title{
Properties of Elementary Fermions of the Standard Model Deduced from Linear Canonical Transformations Representation
}

\section{Ravo Tokiniaina Ranaivoson, Raoelina Andriambololona, Hanitriarivo Rakotoson, Wilfrid Chrysante Solofoarisina}

Information Technology and Theoretical Physics Department, National Institute for Nuclear Science and Technology (INSTN-Madagascar), Antananarivo, Madagascar

\section{Email address:}

tokiniainaravor13@gmail.com (R. T. Ranaivoson), tokhiniaina@gmail.com (R. T. Ranaivoson),

raoelina.andriambololona@gmail.com (R. Andriambololona), raoelinasp@yahoo.fr (R. Andriambololona), jacquelineraoelina@hotmail.com (R. Andriambololona),infotsara@gmail.com (H. Rakotoson), solofoarisinaw@blueline.mg (W. C. Solofoarisina)

\section{To cite this article:}

Ravo Tokiniaina Ranaivoson, Raoelina Andriambololona, Hanitriarivo Rakotoson, Wilfrid Chrysante Solofoarisina. Properties of Elementary Fermions of the Standard Model Deduced from Linear Canonical Transformations Representation. International Journal of Applied Mathematics and Theoretical Physics. Vol. 6, No. 1, 2020, pp. 1-6. doi: 10.11648/j.ijamtp.20200601.11

Received: January 30, 2020; Accepted: February 14, 2020; Published: February 26, 2020

\begin{abstract}
This paper is a continuation of our works concerning Linear Canonical Transformations (LCT) and Phase Space Representation of Quantum Theory. The purpose is to study the spinorial representation of some particular LCT called Isodispersion LCT (ILCT) and to deduce a relation between them and some properties of the elementary fermions of the Standard Model of Particle Physics. After giving the definition of ILCT for the case of a general pseudo-Euclidean space and constructing their spinorial representation, we consider the particular case of a pentadimensional space with signature $(1,4)$. We then deduce a classification of quarks, leptons and their antiparticles according to the values of electric charge, weak hypercharge, weak isospin and colors after the introduction of appropriate operators defined from the generators of the Clifford Algebra corresponding to the ILCT spinorial representation. It is established that the electric charge is composed of four terms, the weak hypercharge of five terms and the weak isospin of two terms. Existence of sterile neutrinos and the possibility of describing a fermions generation with a single field are suggested.
\end{abstract}

Keywords: Linear Canonical Transformations, Spinorial Representation, Quarks, Leptons, Standard Model

\section{Introduction}

In our previous works [1-8], we have performed series of studies on a phase space representation of quantum theory and Linear Canonical Transformations (LCTs). One of the results obtained is the conception of a spinorial representation of the LCTs $[6,8]$. In the present works, our purpose is to highlight a relation which can be established between the LCTs and the elementary fermions of the Standard Model of Particle Physics [9-14] i.e. quarks and leptons. In this regard, we consider a particular case of LCTs called Isodispersion Linear Canonical Transformations (ILCTs).

First, we give the definition of the ILCTs and study their spinorial representation in the case of the $\mathrm{N}$-dimensional pseudo- euclidian space with signature $\left(N_{+}, N_{-}\right)$. Then, we apply this study to a pentadimensional pseudo- euclidian space, signature $(1,4)$. We find that it permits to describe the properties of the fermions belonging to a single generation of the Standard Model and their antiparticles. This pentadimensional space may be considered as the ordinary Minkowski space corresponding to relativistic spacetime, signature $(1,3)$, with an additional fifth dimension. It may be expected that this fifth dimension is related to mass. The choice of the number five for the dimension is related to the fact that the total numbers of particles and antiparticles for a single generation is equal to $2^{5}=32$ (table 1). The introduction of a right-handed (sterile) neutrino and its antiparticle is suggested by our study to complete this list of 32 particles and antiparticles.

Our method may be considered as an extension of the 
approach developed by Zenczykowski for nonrelativistic phase space [15-16]. He obtains that the weak hypercharge is the sum of three terms. In our case, it is composed of five terms (see relation 18).

As in our previous works [1-8], we use for tensorial and matricial calculations the notations developed in the reference [17].

\section{Spinorial Representation of Isodispersion Linear Canonical Transformations}

\subsection{Isodispersion Linear Canonical Transformations}

We have established that LCTs corresponding to a $N$ dimension pseudo-euclidian space $E_{N}$ with signature $\left(N_{+}, N_{-}\right)$ can be defined as the linear transformations which leave invariant the canonical commutation relation defining coordinate and momentum operators $[5,6,8]$. They can be described with the elements of the pseudo-symplectic group $S p\left(2 N_{+}, 2 N_{-}\right)$. In the reference [6], we have introduced the following parameterization for an LCT

$$
\left(\begin{array}{ll}
p^{\prime} & x^{\prime}
\end{array}\right)=\left(\begin{array}{ll}
\not & \mathscr{X}
\end{array}\right) e^{\left(\begin{array}{cc}
\lambda+\mu & \varphi-\theta \\
\varphi+\theta & \lambda-\mu
\end{array}\right)}
$$

in which $\not$ and $\mathscr{*}$ are the $1 \times N$ matrices which admit as elements the components $\nexists_{\mu}$ and $\mathscr{*}_{\mu}(\mu=0, \ldots, N-1)$ of the reduced momentum and coordinate operators $[5,6]$. $\lambda, \mu, \varphi, \theta$ are $N \times N$ matrices verifying the relations

$$
\left\{\begin{array}{c}
\theta^{T}=\eta^{T} \theta \eta=\eta \theta \eta \\
\varphi^{T}=\eta^{T} \varphi \eta=\eta \varphi \eta \\
\mu^{T}=\eta^{T} \mu \eta=\eta \mu \eta \\
\lambda^{T}=-\eta^{T} \lambda \eta=-\eta \lambda \eta \\
\operatorname{spur}(\lambda)=0
\end{array}\right.
$$

$\eta$ being the $N \times N$ matrices admitting as elements the components $\eta_{\mu \nu}$ of the symmetric bilinear form defining the inner product on the pseudo-euclidian space $E_{N}$.

$$
\eta_{\mu \nu}=\left\{\begin{array}{c}
1 \text { if } \mu=v=0,1, \ldots, N_{+}-1 \\
-1 \text { if } \mu=v=N_{+}, N_{+}+1, \ldots, N-1 \\
0 \text { if } \mu \neq v
\end{array}\right.
$$

The infinitesimal form of the LCT in relation (1) is

$$
\left(\begin{array}{ll}
\boldsymbol{P}^{\prime} & \boldsymbol{X}^{\prime}
\end{array}\right)=\left(\begin{array}{ll}
\not{P} & \mathscr{X}
\end{array}\right)\left[1+\left(\begin{array}{ll}
\lambda+\mu & \varphi-\theta \\
\varphi+\theta & \lambda-\mu
\end{array}\right)\right]
$$

For $\mu=0$ and $\varphi=0$, we have

$$
\left(\begin{array}{cc}
\lambda+\mu & \varphi-\theta \\
\varphi+\theta & \lambda-\mu
\end{array}\right)=\left(\begin{array}{cc}
\lambda & -\theta \\
\theta & \lambda
\end{array}\right)
$$

The following relations can be deduced from relations (2)

$$
\left\{\begin{array}{c}
\left(\begin{array}{cc}
\lambda & -\theta \\
\theta & \lambda
\end{array}\right)^{T}=-\left(\begin{array}{cc}
\eta & 0 \\
0 & \eta
\end{array}\right)\left(\begin{array}{cc}
\lambda & -\theta \\
\theta & \lambda
\end{array}\right)\left(\begin{array}{ll}
\eta & 0 \\
0 & \eta
\end{array}\right) \\
\operatorname{Spur}\left(\begin{array}{cc}
\lambda & -\theta \\
\theta & \lambda
\end{array}\right)=0
\end{array}\right.
$$

The relations in (2) means that $\left(\begin{array}{cc}\lambda & -\theta \\ \theta & \lambda\end{array}\right)$ is an element of the Lie algebra $\mathfrak{s D}\left(2 N_{+}, 2 N_{-}\right)$of the pseudo-special orthogonal group $S O\left(2 N_{+}, 2 N_{-}\right)$i.e. the matrix $e^{\left(\begin{array}{cc}\lambda & -\theta \\ \theta & \lambda\end{array}\right)}$ belongs to $S O\left(2 N_{+}, 2 N_{-}\right)$

$$
\left\{\begin{array}{c}
{\left[e^{\left(\begin{array}{cc}
\lambda & -\theta \\
\theta & \lambda
\end{array}\right)}\right]^{T}\left(\begin{array}{cc}
\eta & 0 \\
0 & \eta
\end{array}\right) e^{\left(\begin{array}{cc}
\lambda & -\theta \\
\theta & \lambda
\end{array}\right)}=\left(\begin{array}{ll}
\eta & 0 \\
0 & \eta
\end{array}\right)} \\
\operatorname{Det}\left[e^{\left(\begin{array}{cc}
\lambda & -\theta \\
\theta & \lambda
\end{array}\right)}\right]=1
\end{array}\right.
$$

The relations (1), (5) and (6) imply that the LCT corresponding to $e^{\left(\begin{array}{cc}\lambda & -\theta \\ \theta & \lambda\end{array}\right)}$ leaves invariant the operator

$$
\beth^{+}=\eta^{\mu v} \beth_{\mu \nu}^{+}=\frac{1}{4} \eta^{\mu v}\left(\not_{\mu} \not_{\nu}+\varkappa_{\mu} \mathfrak{x}_{v}\right)
$$

The $\beth_{\mu \nu}^{+}$are the reduced dispersion-codispersion operators introduced in the reference [5]. Explicitly, we have

$$
\left(\begin{array}{ll}
\not^{\prime} & \mathscr{X}^{\prime}
\end{array}\right)=\left(\begin{array}{ll}
\not & \mathscr{X}
\end{array}\right) e^{\left(\begin{array}{cc}
\lambda & -\theta \\
\theta & \lambda
\end{array}\right)} \Leftrightarrow{\beth^{\prime+}}^{+}=\beth^{+}
$$

Relation (7) can be checked using the relations (6) and the relations

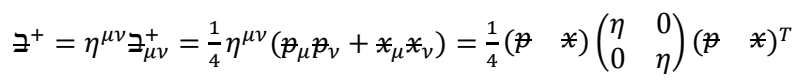

We define the LCTs corresponding to a matrix of the form $e^{\left(\begin{array}{cc}\lambda & -\theta \\ \theta & \lambda\end{array}\right)} \in S O\left(2 N_{+}, 2 N_{-}\right)$as the Isodispersion Linear Canonical Transformations (ILCTs). This definition is in line with the study performed for the case of one dimensional quantum mechanics [3]. As we show in the reference [8], for $\theta=0(\lambda \neq 0)$ an ILCT gives a Lorentz-like transformations. For the case $\lambda=0(\theta \neq 0)$, we have fractional Fourier- like transformations.

\subsection{Spinorial Representations}

As an ILCT is defined with an element $e^{\left(\begin{array}{cc}\lambda & -\theta \\ \theta & \lambda\end{array}\right)}$ of the special pseudo-orthogonal group $S O\left(2 N_{+}, 2 N_{-}\right)$, it can be also represented spinoriarly with an element $\mathcal{S}$ of the spin group $\operatorname{Spin}\left(2 N_{+}, 2 N_{-}\right)$which is the double cover of $S O\left(2 N_{+}, 2 N_{-}\right)$. To construct this spinorial representation, we introduce the operator [6]

$$
\mathbb{P}=\alpha^{\mu} p_{\mu}+\beta^{\mu} \mathscr{x}_{\mu}
$$

in which $\alpha^{\mu}$ and $\beta^{\mu}$ are the generators of the Clifford algebra $\mathfrak{C}\left(2 N_{+}, 2 N_{-}\right)$i.e. they verify the following anticommutation relations:

$$
\left\{\begin{array}{c}
\alpha^{\mu} \alpha^{v}+\alpha^{v} \alpha^{\mu}=2 \eta^{\mu \nu} \\
\beta^{\mu} \beta^{v}+\beta^{v} \beta^{\mu}=2 \eta^{\mu \nu} \\
\alpha^{\mu} \beta^{v}+\beta^{v} \alpha^{\mu}=0
\end{array}\right.
$$

The spinorial representation can be defined explicitly by a mapping $\varrho$, from $S O\left(2 N_{+}, 2 N_{-}\right)$to $\operatorname{Spin}\left(2 N_{+}, 2 N_{-}\right)$, which associates $e^{\left(\begin{array}{cc}\lambda & -\theta \\ \theta & \lambda\end{array}\right)}$ to an element $\mathcal{S}$ of $\operatorname{Spin}\left(2 N_{+}, 2 N_{-}\right)$: 


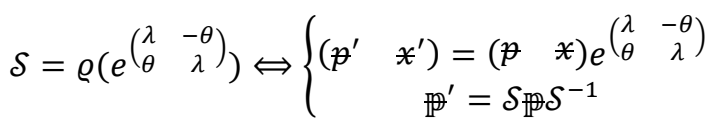

$$
\begin{aligned}
& \mathcal{S}=e^{\left[\frac{1}{4}\left(\eta_{\mu \rho} \lambda_{\nu}^{\rho}+\eta_{\nu \rho} \lambda_{\mu}^{\rho}\right)\left(\alpha^{\mu} \alpha^{\nu}+\beta^{\mu} \beta^{\nu}\right)+\frac{1}{2} \eta_{\mu \rho} \theta_{\nu}^{\rho} \alpha^{\mu} \beta^{\nu}\right]}
\end{aligned}
$$

The operator $\mathcal{S}$ acts on the elements $\vec{\psi}$ of a spinor space $\mathbb{S}$

$$
\vec{\psi}^{\prime}=\mathcal{S} \vec{\psi} \Leftrightarrow \psi^{\prime a}=\mathcal{S}_{b}^{a} \psi^{b}
$$

The couple $(\mathbb{S}, \varrho)$ define the spinorial representation of an ILCT, $\mathbb{S}$ being the representation space and $\varrho$ the group morphism.

\section{Deduction of the Properties of the Elementary Fermions}

We consider the case of a pentadimensional pseudoEuclidian space with signature $(1,4)$. The Clifford algebra and spin group corresponding to the spinorial representation of ILCTs are then respectively $\mathfrak{C}(2,8)$ and Spin $(2,8)$. From the generators $\alpha^{\mu}$ and $\beta^{\mu}$ of the Clifford algebra $\mathfrak{C}(2,8)$, we define the operators

$$
\left\{\begin{array}{l}
y^{0}=\frac{i}{4}\left[\alpha^{0}, \beta^{0}\right]=\frac{1}{2} i \alpha^{0} \beta^{0} \\
y^{1}=\frac{i}{6}\left[\alpha^{1}, \beta^{1}\right]=\frac{1}{3} i \alpha^{1} \beta^{1} \\
y^{2}=\frac{i}{6}\left[\alpha^{2}, \beta^{2}\right]=\frac{1}{3} i \alpha^{2} \beta^{2} \\
y^{3}=\frac{i}{6}\left[\alpha^{3}, \beta^{3}\right]=\frac{1}{3} i \alpha^{3} \beta^{3} \\
y^{4}=\frac{i}{4}\left[\alpha^{4}, \beta^{4}\right]=\frac{1}{2} i \alpha^{4} \beta^{4}
\end{array}\right.
$$

The operators $y^{\mu}$ satisfy the following properties

$$
\left\{\begin{array}{c}
\left(y^{0}\right)^{2}=\left(y^{4}\right)^{2}=\frac{1}{4} \quad\left(y^{1}\right)^{2}=\left(y^{2}\right)^{2}=\left(y^{3}\right)^{2}=\frac{1}{9} \\
{\left[y^{\mu}, y^{v}\right]=y^{\mu} y^{v}-y^{v} y^{\mu}=0}
\end{array}\right.
$$

i. The eigenvalues of $y^{0}$ and $y^{4}$ are $-\frac{1}{2}$ and $\frac{1}{2}$

ii. The eigenvalues of $\mathcal{Y}^{1}, y^{2}, y^{3}$ are $-\frac{1}{3}$ and $\frac{1}{3}$

iii. These operators commute and are simultaneously diagonalizable

These results can be directly and easily checked if we choose appropriate matrices representation of the $\alpha^{\mu}$ and $\beta^{\mu}$. Let us choose

$$
\left\{\begin{array}{c}
\alpha^{0}=\sigma^{1} \otimes \sigma^{0} \otimes \sigma^{0} \otimes \sigma^{0} \otimes \sigma^{0} \\
\alpha^{1}=i \sigma^{3} \otimes \sigma^{1} \otimes \sigma^{0} \otimes \sigma^{0} \otimes \sigma^{0} \\
\alpha^{2}=i \sigma^{3} \otimes \sigma^{3} \otimes \sigma^{1} \otimes \sigma^{0} \otimes \sigma^{0} \\
\alpha^{3}=i \sigma^{3} \otimes \sigma^{3} \otimes \sigma^{3} \otimes \sigma^{1} \otimes \sigma^{0} \\
\alpha^{4}=i \sigma^{3} \otimes \sigma^{3} \otimes \sigma^{3} \otimes \sigma^{3} \otimes \sigma^{1} \\
\beta^{0}=\sigma^{2} \otimes \sigma^{0} \otimes \sigma^{0} \otimes \sigma^{0} \otimes \sigma^{0} \\
\beta^{1}=-i \sigma^{3} \otimes \sigma^{2} \otimes \sigma^{0} \otimes \sigma^{0} \otimes \sigma^{0} \\
\beta^{2}=-i \sigma^{3} \otimes \sigma^{3} \otimes \sigma^{2} \otimes \sigma^{0} \otimes \sigma^{0} \\
\beta^{3}=-i \sigma^{3} \otimes \sigma^{3} \otimes \sigma^{3} \otimes \sigma^{2} \otimes \sigma^{0} \\
\beta^{4}=-i \sigma^{3} \otimes \sigma^{3} \otimes \sigma^{3} \otimes \sigma^{3} \otimes \sigma^{2}
\end{array}\right.
$$

in which $\sigma^{0}$ is the $2 \times 2$ identity matrix and $\sigma^{1}, \sigma^{2}, \sigma^{3}$ are the Pauli matrices

$\sigma^{0}=\left(\begin{array}{ll}1 & 0 \\ 0 & 1\end{array}\right) \quad \sigma^{1}=\left(\begin{array}{ll}0 & 1 \\ 1 & 0\end{array}\right) \quad \sigma^{2}=\left(\begin{array}{cc}0 & -i \\ i & 0\end{array}\right) \quad \sigma^{3}=\left(\begin{array}{cc}1 & 0 \\ 0 & -1\end{array}\right)$

The operators $y^{\mu}$ are represented by the following diagonal matrices

$$
\left\{\begin{array}{l}
y^{0}=-\frac{1}{2} \sigma^{3} \otimes \sigma^{0} \otimes \sigma^{0} \otimes \sigma^{0} \otimes \sigma^{0} \\
y^{1}=-\frac{1}{3} \sigma^{0} \otimes \sigma^{3} \otimes \sigma^{0} \otimes \sigma^{0} \otimes \sigma^{0} \\
y^{2}=-\frac{1}{3} \sigma^{0} \otimes \sigma^{0} \otimes \sigma^{3} \otimes \sigma^{0} \otimes \sigma^{0} \\
y^{3}=-\frac{1}{3} \sigma^{0} \otimes \sigma^{0} \otimes \sigma^{0} \otimes \sigma^{3} \otimes \sigma^{0} \\
y^{4}=-\frac{1}{2} \sigma^{0} \otimes \sigma^{0} \otimes \sigma^{0} \otimes \sigma^{0} \otimes \sigma^{3}
\end{array}\right.
$$

Let us now define the following operators

$$
\left\{\begin{array}{c}
I_{3}=\frac{1}{2} y^{0}-\frac{1}{2} y^{4} \\
Y_{W}=y^{0}+y^{1}+y^{2}+y^{3}+y^{4} \\
Q=y^{0}+\frac{1}{2} y^{1}+\frac{1}{2} y^{2}+\frac{1}{2} y^{3}
\end{array}\right.
$$

As it is seen in the Table 1, these three operators correspond respectively to the electric charge, the weak hypercharge and weak isospin of quarks and leptons belonging to a fermions generation of the standard model and their antiparticles. It may be checked easily that we have the relation

$$
Q=I_{3}+\frac{Y_{W}}{2}
$$

The Table 1 corresponds to a single fermions generation. The example of the first generation is considered: $e_{L}$ is the left-handed negaton and $\overline{e_{L}}$ is its antiparticle (positon). $e_{R}$ is the right-handed negaton and $\overline{e_{R}}$ its antiparticle, $v_{L}$ and $\overline{v_{L}}$ are respectively the left-handed neutrino and its antiparticle. As it is well known, there is no right-handed (sterile) neutrino, $v_{R}$, in the Standard Model but their existence is suggested by this table 1 .

The up and down quarks are respectively denoted $u$ and $d$ and their antiparticles $\bar{u}$ and $\bar{d}$. The lower script refers to chirality ( $\mathrm{R}$ for right- handed and $\mathrm{L}$ for left-handed) and the upper script are colors (blue, green or red).

The existence of the three possible quarks colors is described by the combinations of the eigenvalues of the operators $y^{1}, y^{2}$ and $y^{3}$.

\section{Description of a Fermions generation with a Single Field}

The Table 1 suggests the possibility of describing a fermions generation with a single spinor field $\vec{\psi}$ having 32 components. If we denote $\mathbb{S}$ the spinor space to which belongs the spinor $\vec{\psi}$, we may write:

$$
\mathbb{S}=\mathbb{S}_{e} \oplus \mathbb{S}_{v} \oplus \mathbb{S}_{u} \oplus \mathbb{S}_{d}
$$

in which: $\mathbb{S}_{e}$ is the subspace of $\mathbb{S}$ composed by the electron- 
type field, $\mathbb{S}_{v}$ the subspace of neutrino-type field, $\mathbb{S}_{u}$ the subspace of up quark-type field and $\mathbb{S}_{d}$ the subspace of quark down-type field. Explicitly, we have for a general field $\vec{\psi}$ itself

$\vec{\psi}=\psi^{a} \vec{\zeta}_{a}=\vec{\psi}_{e}+\vec{\psi}_{v}+\vec{\psi}_{u}+\vec{\psi}_{d}=\left(\Pi_{e}+\Pi_{v}+\Pi_{u}+\Pi_{d}\right) \vec{\psi}$

in which $\left\{\vec{\zeta}_{a}\right\},(a=1$ to 32$)$, is a basis of $\mathbb{S}$ constituted by the eigenspinors $\vec{\zeta}_{a}$ of the operators
$\mathcal{Y}^{0}, \mathcal{Y}^{1}, \mathcal{Y}^{2}, \mathcal{Y}^{3}, \mathcal{Y}^{4}, I_{3}, Y_{W}$ and $Q . \vec{\psi}_{e}, \vec{\psi}_{v}, \vec{\psi}_{u}$ and $\vec{\psi}_{d}$ are respectively the electron-type field $\left(\vec{\psi}_{e} \in \mathbb{S}_{e}\right)$, neutrino-type field a $\left(\vec{\psi}_{v} \in \mathbb{S}_{v}\right)$, up quark-type field $\left(\vec{\psi}_{u} \in \mathbb{S}_{u}\right)$ and down quark-type field $\left(\vec{\psi}_{d} \in \mathbb{S}_{d}\right) . \Pi_{e}, \Pi_{v}, \Pi_{u}$ and $\Pi_{d}$ are the projection operators corresponding to the subspaces $\mathbb{S}_{e}, \mathbb{S}_{v}, \mathbb{S}_{u}$ and $\mathbb{S}_{d}$. These projection operators can be expressed as polynomials of $\mathcal{Y}^{0}, \mathcal{Y}^{1}, \mathcal{Y}^{2}$ and $\mathcal{Y}^{3}$.

Table 1. Classification of quarks, leptons (and their antiparticles) belonging to a generation according to the eigenvalues of the operators $y^{0}, y^{1}, y^{2}, y^{3}, y^{4}$, $I_{3}, Y_{W}$ and $Q$.

\begin{tabular}{|c|c|c|c|c|c|c|c|c|c|}
\hline $\mathbf{N}^{\circ}$ & $y^{0}$ & $y^{1}$ & $y^{2}$ & $y^{3}$ & $y^{4}$ & $I_{3}$ & $Y_{W}$ & $Q$ & Particle \\
\hline 1 & $-1 / 2$ & $-1 / 3$ & $-1 / 3$ & $-1 / 3$ & $-1 / 2$ & 0 & -2 & -1 & $e_{R}$ \\
\hline 2 & $-1 / 2$ & $-1 / 3$ & $-1 / 3$ & $-1 / 3$ & $1 / 2$ & $-1 / 2$ & -1 & -1 & $e_{L}$ \\
\hline 3 & $-1 / 2$ & $-1 / 3$ & $-1 / 3$ & $1 / 3$ & $-1 / 2$ & 0 & $-4 / 3$ & $-2 / 3$ & $\bar{u}_{R}^{\text {blue }}$ \\
\hline 4 & $-1 / 2$ & $-1 / 3$ & $-1 / 3$ & $1 / 3$ & $1 / 2$ & $-1 / 2$ & $-1 / 3$ & $-2 / 3$ & $\bar{u}_{L}^{\text {blue }}$ \\
\hline 5 & $-1 / 2$ & $-1 / 3$ & $1 / 3$ & $-1 / 3$ & $-1 / 2$ & 0 & $-4 / 3$ & $-2 / 3$ & $\bar{u}_{R}^{\text {green }}$ \\
\hline 6 & $-1 / 2$ & $-1 / 3$ & $1 / 3$ & $-1 / 3$ & $1 / 2$ & $-1 / 2$ & $-1 / 3$ & $-2 / 3$ & $\bar{u}_{L}^{\text {green }}$ \\
\hline 7 & $-1 / 2$ & $-1 / 3$ & $1 / 3$ & $1 / 3$ & $-1 / 2$ & 0 & $-2 / 3$ & $-1 / 3$ & $d_{R}^{r e d}$ \\
\hline 8 & $-1 / 2$ & $-1 / 3$ & $1 / 3$ & $1 / 3$ & $1 / 2$ & $-1 / 2$ & $1 / 3$ & $-1 / 3$ & $d_{L}^{\text {red }}$ \\
\hline 9 & $-1 / 2$ & $1 / 3$ & $-1 / 3$ & $-1 / 3$ & $-1 / 2$ & 0 & $-4 / 3$ & $-2 / 3$ & $\bar{u}_{R}^{r e d}$ \\
\hline 10 & $-1 / 2$ & $1 / 3$ & $-1 / 3$ & $-1 / 3$ & $1 / 2$ & $-1 / 2$ & $-1 / 3$ & $-2 / 3$ & $\bar{u}_{L}^{\text {red }}$ \\
\hline 11 & $-1 / 2$ & $1 / 3$ & $-1 / 3$ & $1 / 3$ & $-1 / 2$ & 0 & $-2 / 3$ & $-1 / 3$ & $d_{R}^{\text {green }}$ \\
\hline 12 & $-1 / 2$ & $1 / 3$ & $-1 / 3$ & $1 / 3$ & $1 / 2$ & $-1 / 2$ & $1 / 3$ & $-1 / 3$ & $d_{L}^{\text {green }}$ \\
\hline 13 & $-1 / 2$ & $1 / 3$ & $1 / 3$ & $-1 / 3$ & $-1 / 2$ & 0 & $-2 / 3$ & $-1 / 3$ & $d_{R}^{b l u e}$ \\
\hline 14 & $-1 / 2$ & $1 / 3$ & $1 / 3$ & $-1 / 3$ & $1 / 2$ & $-1 / 2$ & $1 / 3$ & $-1 / 3$ & $d_{L}^{\text {blue }}$ \\
\hline 15 & $-1 / 2$ & $1 / 3$ & $1 / 3$ & $1 / 3$ & $-1 / 2$ & 0 & 0 & 0 & $\bar{v}_{R}$ \\
\hline 16 & $-1 / 2$ & $1 / 3$ & $1 / 3$ & $1 / 3$ & $1 / 2$ & $-1 / 2$ & 1 & 0 & $\bar{v}_{L}$ \\
\hline 17 & $1 / 2$ & $-1 / 3$ & $-1 / 3$ & $-1 / 3$ & $-1 / 2$ & $1 / 2$ & -1 & 0 & $v_{L}$ \\
\hline 18 & $1 / 2$ & $-1 / 3$ & $-1 / 3$ & $-1 / 3$ & $1 / 2$ & 0 & 0 & 0 & $v_{R}$ \\
\hline 19 & $1 / 2$ & $-1 / 3$ & $-1 / 3$ & $1 / 3$ & $-1 / 2$ & $1 / 2$ & $-1 / 3$ & $1 / 3$ & $\bar{d}_{L}^{\text {blue }}$ \\
\hline 20 & $1 / 2$ & $-1 / 3$ & $-1 / 3$ & $1 / 3$ & $1 / 2$ & 0 & $2 / 3$ & $1 / 3$ & $\bar{d}_{R}^{\text {blue }}$ \\
\hline 21 & $1 / 2$ & $-1 / 3$ & $1 / 3$ & $-1 / 3$ & $-1 / 2$ & $1 / 2$ & $-1 / 3$ & $1 / 3$ & $\bar{d}_{L}^{g r e e}$ \\
\hline 22 & $1 / 2$ & $-1 / 3$ & $1 / 3$ & $-1 / 3$ & $1 / 2$ & 0 & $2 / 3$ & $1 / 3$ & $\bar{d}_{R}^{\text {green }}$ \\
\hline 23 & $1 / 2$ & $-1 / 3$ & $1 / 3$ & $1 / 3$ & $-1 / 2$ & $1 / 2$ & $1 / 3$ & $2 / 3$ & $u_{L}^{r e d}$ \\
\hline 24 & $1 / 2$ & $-1 / 3$ & $1 / 3$ & $1 / 3$ & $1 / 2$ & 0 & $-4 / 3$ & $2 / 3$ & $u_{R}^{r e d}$ \\
\hline 25 & $1 / 2$ & $1 / 3$ & $-1 / 3$ & $-1 / 3$ & $-1 / 2$ & $1 / 2$ & $1 / 3$ & $1 / 3$ & $\bar{d}_{L}^{r e d}$ \\
\hline 26 & $1 / 2$ & $1 / 3$ & $-1 / 3$ & $-1 / 3$ & $1 / 2$ & 0 & $2 / 3$ & $1 / 3$ & $\bar{d}_{R}^{\text {red }}$ \\
\hline 27 & $1 / 2$ & $1 / 3$ & $-1 / 3$ & $1 / 3$ & $-1 / 2$ & $1 / 2$ & $1 / 3$ & $2 / 3$ & $u_{L}^{g r e e n}$ \\
\hline 28 & $1 / 2$ & $1 / 3$ & $-1 / 3$ & $1 / 3$ & $1 / 2$ & 0 & $-4 / 3$ & $2 / 3$ & $u_{R}^{g r e e n}$ \\
\hline 29 & $1 / 2$ & $1 / 3$ & $1 / 3$ & $-1 / 3$ & $-1 / 2$ & $1 / 2$ & $1 / 3$ & $2 / 3$ & $u_{L}^{\text {blue }}$ \\
\hline 30 & $1 / 2$ & $1 / 3$ & $1 / 3$ & $-1 / 3$ & $1 / 2$ & 0 & $-4 / 3$ & $2 / 3$ & $u_{R}^{\text {blue }}$ \\
\hline 31 & $1 / 2$ & $1 / 3$ & $1 / 3$ & $1 / 3$ & $-1 / 2$ & $1 / 2$ & 1 & 1 & $\overline{e_{L}}$ \\
\hline 32 & $1 / 2$ & $1 / 3$ & $1 / 3$ & $1 / 3$ & $1 / 2$ & 0 & 2 & 1 & $\overline{e_{R}}$ \\
\hline
\end{tabular}

From the table 1, we can deduce

i. The electron-type field

$$
\begin{gathered}
\vec{\psi}_{e}=\Pi_{e} \vec{\psi}=\psi^{1} \vec{\zeta}_{1}+\psi^{2} \vec{\zeta}_{2}+\psi^{31} \vec{\zeta}_{31}+\psi^{32} \vec{\zeta}_{32} \\
\Pi_{e}=\frac{\left(1-2 y^{0}\right)\left(1-3 y^{1}\right)\left(1-3 y^{2}\right)\left(1-3 y^{3}\right)}{16}+\frac{\left(1+2 y^{0}\right)\left(1+3 y^{1}\right)\left(1+3 y^{2}\right)\left(1+3 y^{3}\right)}{16}
\end{gathered}
$$

ii. The neutrino-type field

$$
\begin{gathered}
\vec{\psi}_{v}=\Pi_{v} \vec{\psi}=\psi^{15} \vec{\zeta}_{15}+\psi^{16} \vec{\zeta}_{16}+\psi^{17} \vec{\zeta}_{17}+\psi^{18} \vec{\zeta}_{18} \\
\Pi_{v}=\frac{\left(1-2 y^{0}\right)\left(1+3 y^{1}\right)\left(1+3 y^{2}\right)\left(1+3 y^{3}\right)}{16}+\frac{\left(1+2 y^{0}\right)\left(1-3 y^{1}\right)\left(1-3 y^{2}\right)\left(1-3 y^{3}\right)}{16}
\end{gathered}
$$

iii. The up quark-type field

$$
\begin{array}{r}
\vec{\psi}_{u}=\Pi_{u} \vec{\psi}=\psi^{3} \vec{\zeta}_{3}+\psi^{4} \vec{\zeta}_{4}+\psi^{5} \vec{\zeta}_{5}+\psi^{6} \vec{\zeta}_{6}+\psi^{9} \vec{\zeta}_{9}+\psi^{10} \vec{\zeta}_{10}+\psi^{23} \vec{\zeta}_{23}+\psi^{24} \vec{\zeta}_{24}+\psi^{27} \vec{\zeta}_{27}+\psi^{28} \vec{\zeta}_{28}+\psi^{29} \vec{\zeta}_{29}+\psi^{30} \vec{\zeta}_{30} \\
\Pi_{u}=\frac{\left(1-2 y^{0}\right)\left(1-3 y^{1}\right)\left(1-3 y^{2}\right)\left(1+3 y^{3}\right)}{16}+\frac{\left(1-2 y^{0}\right)\left(1-3 y^{1}\right)\left(1+3 y^{2}\right)\left(1-3 y^{3}\right)}{16}+\frac{\left(1-2 y^{0}\right)\left(1+3 y^{1}\right)\left(1-3 y^{2}\right)\left(1-3 y^{3}\right)}{16}
\end{array}
$$




$$
+\frac{\left(1+2 y^{0}\right)\left(1-3 y^{1}\right)\left(1+3 y^{2}\right)\left(1+3 y^{3}\right)}{16}+\frac{\left(1+2 y^{0}\right)\left(1+3 y^{1}\right)\left(1-3 y^{2}\right)\left(1+3 y^{3}\right)}{16}+\frac{\left(1+2 y^{0}\right)\left(1+3 y^{1}\right)\left(1+3 y^{2}\right)\left(1-3 y^{3}\right)}{16}
$$

iv. The down quark-type field

$$
\begin{aligned}
& \vec{\psi}_{d}=\Pi_{d} \vec{\psi}= \psi^{7} \vec{\zeta}_{7}+\psi^{8} \vec{\zeta}_{8}+\psi^{11} \vec{\zeta}_{11}+\psi^{12} \vec{\zeta}_{12}+\psi^{13} \vec{\zeta}_{13}+\psi^{14} \vec{\zeta}_{14}+\psi^{19} \vec{\zeta}_{19}, \psi^{20} \vec{\zeta}_{20}+\psi^{21} \vec{\zeta}_{21}+\psi^{22} \vec{\zeta}_{22}+\psi^{25} \vec{\zeta}_{25}+\psi^{26} \vec{\zeta}_{26} \\
& \Pi_{d}=\frac{\left(1-2 y^{0}\right)\left(1-3 y^{1}\right)\left(1+3 y^{2}\right)\left(1+3 y^{3}\right)}{16}+\frac{\left(1-2 y^{0}\right)\left(1+3 y^{1}\right)\left(1-3 y^{2}\right)\left(1+3 y^{3}\right)}{16}+\frac{\left(1-2 y^{0}\right)\left(1+3 y^{1}\right)\left(1+3 y^{2}\right)\left(1-3 y^{3}\right)}{16} \\
&+\frac{\left(1+2 y^{0}\right)\left(1-3 y^{1}\right)\left(1-3 y^{2}\right)\left(1+3 y^{3}\right)}{16}+\frac{\left(1+2 y^{0}\right)\left(1-3 y^{1}\right)\left(1+3 y^{2}\right)\left(1-3 y^{3}\right)}{16}+\frac{\left(1+2 y^{0}\right)\left(1+3 y^{1}\right)\left(1-3 y^{2}\right)\left(1-3 y^{3}\right)}{16}
\end{aligned}
$$

\section{Discussion and Conclusion}

Table 1 shows as expected that it is possible to deduce some properties of the elementary fermions of the Standard Model from the spinorial representation of ILCT corresponding to a pentadimensional pseudo-euclidian space with signature $(1,4)$.

According to the relations (18), the values of electric charges, weak hypercharge, weak isospin and colors charges can be considered as corresponding to the eigenvalues of linear combinations of the operators $y^{\mu}$ defined in the relation (14) from the generators $\alpha^{\mu}$ and $\beta^{\mu}$ of the Clifford algebra $\mathbb{C}(2,8)$.

Our study suggests the existence of right handed (sterile) neutrino. Interests are currently being brought to these particles even on the experimental side [18-20]. According to Table 1, the standard model parameters (weak isospin, weak hypercharge and electric charge) corresponding to these kinds of particles are, as expected, equal to zero.

As discussed in the section 4, our work introduces also the possibility of describing a fermions generation with a single field. The fields corresponding to each type of particles can then be seen as just components of this single field.

This work brings some new point of views concerning the relation between spacetime, momentum, energy and particles. It shows that phase space in quantum theory, linear canonical transformations and particles are deeply linked.

The results may be exploited and extended to build a new theory of particles interactions beyond the Standard Model. It is worth pointing out that LCTs permit at the same time to describe linear mixing of spacetime and momentum-energy and the change of particles flavours.

\section{References}

[1] Ravo Tokiniaina Ranaivoson: Raoelina Andriambololona, Rakotoson Hanitriarivo, Roland Raboanary: Study on a Phase Space Representation of Quantum Theory, arXiv: 1304.1034 [quant-ph], International Journal of Latest Research in Science andTechnology, ISSN (Online): 2278-5299, Vol. 2, Issue 2, pp. 26-35, March-April 2013.

[2] Raoelina Andriambololona, Ravo Tokiniaina Ranaivoson, Rakotoson Hanitriarivo, Victor Harison, Derivation of equations for scalar and fermion fields using properties of dispersion-codispersion operators, arXiv: 1401.6526 [quantph], International Journal of Latest Research in Science and Technology ISSN (Online): 2278-5299, Vol. 3, Issue 1, pp. 4852, January-February 2014.
[3] Raoelina Andriambololona, Ravo Tokiniaina Ranaivoson, Rakotoson Hanitriarivo, Wilfrid Chrysante Solofoarisina: Study on Linear Canonical Transformation in a Framework of a Phase Space Representation of Quantum Mechanics, arXiv: 1503.02449 [quant-ph], International Journal of Applied Mathematics and Theoretical Physics. Vol. 1, No. 1, pp. 1-8, 2015.

[4] Hanitriarivo Rakotoson, Raoelina Andriambololona, Ravo Tokiniaina Ranaivoson, Raboanary Roland, coordinate, momentum and dispersion operators in phase space representation, arXiv: 1707.02223 [quant-ph], International Journal of Latest Research in Science and Technology, ISSN (Online): 2278-5299 Vol. 6, Issue 4, pp. 8-13, July-August 2017.

[5] Raoelina Andriambololona, Ravo Tokiniaina Ranaivoson, Hanitriarivo Rakotoson, Hasimbola Damo Emile Randriamisy, Dispersion Operators Algebra and Linear Canonical Transformations, arXiv: 1608.02268 [quant-ph], International Journal of Theoretical Physics, Vol. 56, Issue 4, pp. 12581273, Springer, April 2017.

[6] Raoelina Andriambololona, Ravo Tokiniaina Ranaivoson, Hanitriarivo Rakotoson, Study on a Spinorial Representation of Linear Canonical Transformation, arXiv: 1711.04975 [quant-ph], International Journal of Applied Mathematics and Theoretical Physics. Vol. 5, No. 3, pp. 58-65, 2019.

[7] Ravo Tokiniaina Ranaivoson, Raoelina Andriambololona, Hanitriarivo Rakotoson, Properties of Phase Space Wavefunctions and Eigenvalue Equation of Momentum Dispersion Operator, arXiv: 1711.07308 [quant-ph], International Journal of Applied Mathematics and Theoretical Physics. Vol. 4, No. 1, 2018, pp. 8-14, 2018.

[8] Hanitriarivo Rakotoson, Raoelina Andriambololona, Ravo Tokiniaina Ranaivoson, Roland Raboanary, Linear Canonical Transformations in Relativistic Quantum Physics, arXiv: 1804.10053 [quant-ph], 2018.

[9] P. Aurenche, The Standard Model of particle physics, arXiv: hep-ph/9712342, 1997.

[10] M. J. Herrero, The Standard Model, arXiv: 0901.0241 [hepph], 1998.

[11] Paul Langacker, Introduction to the Standard Model and Electroweak Physics, arXiv: 0901.0241 [hep-ph], 2009.

[12] S. F. Novaes, Standard Model: An Introduction, arXiv: hep$\mathrm{ph} / 0001283,2000$.

[13] Jean Iliopoulos, Introduction to the Standard Model of the Electro-Weak Interactions, 2012 CERN Summer School of Particle Physics, Angers: France (2012), arXiv: 1305.6779 [hep-ph], 2013. 
[14] Guido Altarelli, The Standard Model of Particle Physics, arXiv: hep-ph/0510281, 2005.

[15] Piotr Zenczykowski, Leptons, quarks, and their antiparticles: a phase-spaceview, arXiv: 0905.1207 [hep-th], Int. J. Theor. Phys. 49: 2246-2262, 2010.

[16] Piotr Zenczykowski, From Clifford Algebra of Nonrelativistic Phase Space to Quarks and Leptons of the Standard Model, arXiv: 1505.03482 [hep-ph], 2015.

[17] Raoelina Andriambololona, Algèbre linéaire et multilinéaire, Collection LIRA, INSTN- Madagascar, 1986.
[18] Marco Drewes, The Phenomenology of Right Handed Neutrinos, arXiv: 1303.6912 [hep-ph], Int. J. Mod. Phys. E, $22,1330019,2013$

[19] J. M. Conrad and M. H. Shaevitz, Sterile Neutrinos: An Introduction to Experiments, arXiv: 1609.07803 [hep-ex], 2017.

[20] S. Böser, C. Buck, C. Giunti, J. Lesgourgues, L. Ludhova, S. Mertens, A. Schukraft, M. Wurm, Status of Light Sterile Neutrino Searches, arXiv: 1906.01739 [hep-ex], Progress in Particle and Nuclear Physics, Volume 111, March 2020. 